\title{
Domain-and species-specific monoclonal antibodies recognize the Von Willebrand Factor-C domain of CCN5
}

\author{
Lan Wei • Frank Mckeon • Joshua W. Russo • \\ Joan Lemire • John Castellot
}

Received: 19 February 2009 / Accepted: 6 April 2009/Published online: 29 April 2009

(C) The Author(s) 2009. This article is published with open access at Springerlink.com

\begin{abstract}
The CCN family of proteins typically consists of four distinct peptide domains: an insulin-like growth factor binding protein-type (IGFBP) domain, a Von Willebrand Factor $\mathrm{C}(\mathrm{VWC})$ domain, a thrombospondin type 1 repeat (TSP1) domain, and a carboxy-terminal (CT) domain. The six family members participate in many processes, including proliferation, motility, cell-matrix signaling, angiogenesis, and wound healing. Accumulating evidence suggests that truncated and alternatively spliced isoforms are responsible for the diverse functions of $\mathrm{CCN}$ proteins in both normal and pathophysiologic states. Analysis of the properties and functions of individual $\mathrm{CCN}$ domains further corroborates this idea. CCN5 is unique among the CCN family members because it lacks the CT-domain. To dissect the domain functions of CCN5, we are developing domain-specific mouse monoclonal antibodies. Monoclonal antibodies have the advantages of great specificity, reproducibility, and ease of long-term storage and production. In this communication, we injected mixtures of GST-fused rat CCN5 domains into mice to generate monoclonal antibodies. To identify the domains recognized by the antibodies, we constructed serial expression plasmids that express dual-tagged rat $\mathrm{CCN} 5$ domains. All of the monoclonal antibodies generated to date recognize the VWC domain, indicating it is the most highly immunogenic of the CCN5 domains. We characterized one particular clone, $22 \mathrm{H} 10$, and found that it recognizes mouse and rat CCN5, but
\end{abstract}

L. Wei $\cdot$ J. W. Russo $\cdot$ J. Lemire $\cdot$ J. Castellot $(\bowtie)$

Department of Anatomy and Cellular Biology,

Tufts University School of Medicine,

136 Harrison Avenue,

Boston, MA 02111, USA

e-mail: john.castellot@tufts.edu

F. Mckeon

Department of Cell Biology, Harvard Medical School,

200 Longwood Avenue, SGM 408, Boston, MA 02115, USA not human recombinant CCN5. Purified 22H10 was successfully applied in Western Blot analysis, immunofluorescence of cultured cells and tissues, and immunoprecipitation, indicating that it will be a useful tool for domain analysis and studies of mouse-human tumor models.

Keywords Mouse monoclonal antibody $\cdot \mathrm{CCN}$ family . CCN5 - WISP2 - VWC domain · Dual-tagged domain constructs

\begin{tabular}{ll}
\multicolumn{2}{l}{ Abbreviations } \\
IGFBP & insulin-like growth factor binding module \\
VWC & Von Willebrand Factor C module \\
TSP & Thrombospondin type 1 repeat \\
I & IGFBP domain \\
V & VWC domain \\
T & TSP1 domain
\end{tabular}

\section{Introduction}

Members of the CCN family of proteins play a significant role in a variety of critical cellular processes including cell adhesion, migration, proliferation, gene expression, differentiation, and survival (Bleau et al. 2005; Kubota and Takigawa 2007; Perbal 2004). CCN proteins fill this role, at least in part, via interactions with extracellular matrix components and growth factors (Chen and Lau 2008). With the exception of CCN5, CCN proteins are defined by their four modular domains: the insulin-like growth factor binding protein-like domain (IGFBP), a Von Willebrand factor C-like domain (VWC), a Thrombospondin 1 repeat domain (TSP-1) and a C-terminal cysteine knot (CT). CCN5 is unique among the family members in that it lacks the $\mathrm{CT}$ domain. 
Understanding the functional properties of individual domains has yielded important insights into mechanism. For example, two $\mathrm{CCN}$ proteins - $\mathrm{CCN} 1$ and $\mathrm{CCN} 2-$ bind to integrins through the TSP-1/CT and VWC domains, and appear to regulate a diverse array of biological functions, including fibroblast adhesion, angiogenesis and MAPK activation (Chen et al. 2004; Leu et al. 2004; Leu et al. 2003). Deletion of the CT domain abrogates the ability of CCN3 to induce Notch signaling and inhibits osteogenic differentiation and proliferation (Katsuki et al. 2008). In addition, a naturally occurring isoform of CCN3 lacking the TSP-1 domain relocates from the nucleus to the cytoplasm of tumor cells in the renal neoplasm, Wilm's tumor (Subramaniam et al. 2008). Recently, we discovered differentially expressed isoforms of CCN5; exploration of the structure-function relationships is now underway.

These studies, among others, indicate the importance of studying CCN proteins not only at the level of the full-length protein, but also at the level of the constituent domains. The interaction and availability of ligand/receptors for each individual domain, and the expression $\mathrm{CCN}$ variants via mRNA splicing and/or post-translational modifications, may help explain the diverse functions of $\mathrm{CCN}$ proteins in different cells and tissues (de Winter et al. 2008; Holbourn et al. 2008; Rachfal and Brigstock 2005).

CCN5 is a unique member of the $\mathrm{CCN}$ family in that it is the only family member lacking the C-terminal domain (Delmolino et al. 2001; Pennica et al. 1998; Zhang et al. 1998). Altered CCN5 expression levels are associated with a number of disease states including arterial restenosis, uterine leiomyoma, and breast carcinoma (Banerjee et al. 2008; Lake and Castellot 2003; Mason et al. 2004b). There is also the suggestion that CCN5 expression is critical in development, as transgenic pan-overexpression or pan-knockdown of CCN5 in mice consistently results in embryonic lethality (unpublished finding). Furthermore, studies utilizing adenoviral overexpression of CCN5 in vascular and uterine smooth muscle cells have shown inhibition of SMC proliferation and motility, both in vitro and in animal models, underscoring the promise of this protein in future therapeutic uses (Lake et al. 2003; Mason et al. 2004b) Jones et al. 2007).

The availability of antibodies that recognize specific epitopes within individual domains of CCN5 would be a valuable tool for studying the structure-function relationship of the three peptide domains of CCN5. Currently, the antibodies used to detect $\mathrm{CCNs}$ are either affinity purified rabbit polyclonal antibodies raised against peptide fragments of CCN proteins, or rabbit polyclonal antibodies raised against recombinant CCN proteins (Brigstock et al. 1997; Chevalier et al. 1998; Kutz et al. 2005; Lake et al. 2003; Steffen et al. 1998; Yang and Lau 1991; Zoubine et al. 2001). These antibodies have proven highly useful in monitoring full length $\mathrm{CCN}$ protein, but they are limited in their ability to define the presence of individual domains (in the case of polyclonal antibodies raised against peptide fragments), or lack domain specificity (in the case of those raised against recombinant protein). A clever alternative approach was used by Perbal group, in which polyclonal antibodies were raised against each domain of $\mathrm{CCN} 3$. These antibodies were then used to define CCN3 isoform expression in a number of different cancer samples (Lazar et al. 2007). We are aware of only one report using monoclonal antibodies: Tamatani et al used partially purified recombinant CCN2 to generate monoclonal antibodies against CCN2 (Tamatani et al. 1998).

In this paper, we report our efforts to develop monoclonal antibodies to the three domains of CCN5. To date, all of the positive hybridoma clones isolated recognize the VWC domain. Characterization of one of these antibodies, $22 \mathrm{H} 10$, indicates that it is a useful antibody for immunoblotting, immunofluorescence microscopy, and immunoprecipitation. The high degree of specificity, reproducibility, and ease of producing large quantities of monoclonal antibodies should make this approach a useful one for domain analysis and other mechanistic studies.

\section{Materials and methods}

\section{Cell culture}

All cell were cultured at $37^{\circ} \mathrm{C}$ in a humidified, $5 \% \mathrm{CO}_{2} /$ 95\% air atmosphere. Sprague-Dawley aorta smooth muscle cells were cultured using high glucose RPMI 1640 medium (GIBCO) containing 10\% bovine growth serum (BGS, Hyclone), $2 \mathrm{mM}$ L-glutamine (GIBCO), and 100ug/ml penicillin/ streptomycin (GIBCO). BHK and $3 \mathrm{~T} 3$ cells were cultured in high glucose DMEM (GIBCO) containing 10\% BGS, L-glutamine, and penicillin/streptomycin. Hybridoma clones were cultured in HAT hybridoma selection media containing DMEM, 25\% heat inactivated serum (Sigma CPSR3), L-glutamine, penicillin/ streptomycin, HAT supplement solution (hypoxanthine, aminopterin, thymidine; Invitrogen), 7.8\% NCTC-109 media (GIBCO), nonessential amino acids (Hyclone). HT media is complete DMEM containing HT supplement solution (hypoxanthine, thymidine; Invitrogen). HI-DMEM media is same as complete DMEM except that it contains heat-inactivated fetal bovine serum (FBS, Hyclone). B-27 media is basal DMEM with L-glutamine, penicillin/streptomycin, and B-27 supplement (GIBCO).

Sprague-Dawley rat aorta smooth muscle (SDSM) cells were isolated as previously described (Lake et al. 2003). SDSM were used at passage 8 or lower. Growth-arrest of SDSM cells was accomplished by culturing cells for $72-96 \mathrm{~h}$ in RPMI containing only $0.4 \%$ serum plus L-glutamine, and penicillin/streptomycin. Selected hybrid- 
omas were first grown in HT media for subcloning through limited dilution, then grown in HI-DMEM media. Stock cultures were frozen in HI-DMEM with $10 \%$ dimethyl sulfoxide (DMSO, SIGMA).

Construction of CCN5 expression plasmids

\section{Eukaryotic expression plasmids for recombinant human, mouse, and rat CCN5}

Human, mouse CCN5 ORFs were PCR amplified from IMAGE clones purchased from Open Biosystems (human: MHS1011-7509651; mouse: MMM1013-7510036). Rat CCN5 ORF was PCR amplified from cDNA samples derived from growth-arrested SDSM cells. The primers are listed as pair 9 and 10 in Table 1. Gene cloning was accomplished using the Gateway System (Invitrogen, K2400-20). Briefly, PCR fragments of CCN5 ORFs were inserted into entry vector $\mathrm{pENTR/D-TOPO}$. After sequence verification, the inserts were switched into the destiny vector pcDNADEST40 for eukaryotic cell expression. The recombinant CCN5 proteins were tagged at the C-terminus with one V5 epitope and six histidines. The recombinant $\mathrm{CCN} 5$ proteins utilized the endogenous CCN5 signal peptide for secretion.

\section{Eukaryotic expression plasmids for dual-tagged rat CCN5 domains}

Each of the rat CCN5 domains and domain combinations were PCR amplified from the rat entry plasmid cloned above. Primer pairs used for cloning are indicated in Fig. 1. Primer sequences and $\mathrm{XbaI} / \mathrm{HindIII}$ linkers are shown in Table 1. To make the IT domain, an XhoI site was added in frame. All PCR fragments were cloned into pCR2.1-TOPO vector
(Invitrogen, 45-0641) for sequencing and further subcloning. The DNA of each domain was excised with XbaI and HindIII, and then ligated into the expression vector pflag-myc-CMV 20 (SIGMA, E5526). As shown in Table 2, the resulting proteins all have a flag tag (DYKDDDDK) in the N-terminus and a c-myc tag (EQKLISEEDL) in the $\mathrm{C}$-terminus. These plasmids were used to screen for positive hybridoma clones and for mapping the epitope of anti-CCN5 antibodies.

\section{Bacterial expression plasmids for GST-fused CCN5 domains}

The rat CCN5 domains I, V, T, IV and IVT in pCR2.1TOPO vectors were sub-cloned in-frame into pGEX-4T-1 vector (Amersham-GE Healthcare, 27-4580-01) between the EcoRI and XhoI restriction sites.

\section{Bacterial expression of GST-fusion proteins}

High-level bacterial expression of GST-fused proteins and inclusion body isolation followed the protocol described by Shi et al. (Shi et al. 2008). Briefly, transformed protease-deficient E. coli host strain BL21 (DE3) (Novagen) clones were selected by ampicillin, and then cultured in $37^{\circ} \mathrm{C}$ until the O.D. was 0.6 units. GSTfusion protein expression was further induced by culturing for $4 \mathrm{~h}$ in media containing $0.5 \mathrm{mM}$ isopropyl- $\beta$-dthiogalactoside (IPTG). The bacterial pellet was lysed by several rounds of sonication in PBS followed by centrifugation at $5000 \mathrm{xg}$ for $5 \mathrm{~min}$ to pellet the inclusion bodies. The purified inclusion bodies were resuspended in equal amount of PBS and a small portion of was analyzed by SDS-PAGE.
Table 1 Sequences of primers shown in Fig. 1

\begin{tabular}{llll}
\hline Primer ID & Direction & Linker & Sequence \\
\hline 1 & Forward & HindIII & AAGCTT GTGTGTGCCCAGCTGTGCCG \\
2 & Reverse & XbaI & TCTAGA GAGACACACAGCCCCATGGCCG \\
3 & Forward & HindIII & AAGCTT TTGGATGAGGATGACGGTAGC \\
4 & Reverse & XbaI & TCTAGA TTGCGCCGCGGAGCGCTGGAT \\
5 & Forward & HindIII & AAGCTT GGACACCAACTTTCTGCCCT \\
6 & Reverse & XbaI & TCTAGA GAAAGCACTGTTCCATGAGCTG \\
7 & Reverse & XhoI & CTCGAG GAGACACACAGCCCCATGGCCG \\
8 & Forward & XhoI & CTCGAG GGACACCAACTTTCTGCCCT \\
9 human & Forward & CACC & CACC ATGAGAGGCACACCGAAGAC \\
10 human & Reverse & N/A & GAAGGCACTGTTTGTGGACT \\
9 mouse & Forward & CACC & CACC ATGAGGGGCAACCCACTG \\
10 mouse & Reverse & N/A & GAAGGCACTGTTCCATGAGC \\
9 rat & Forward & CACC & CACC ATGAGGGGCAGCCCACT \\
10 rat & Reverse & N/A & GAAAGCACTGTTCCATGAGC \\
\hline
\end{tabular}




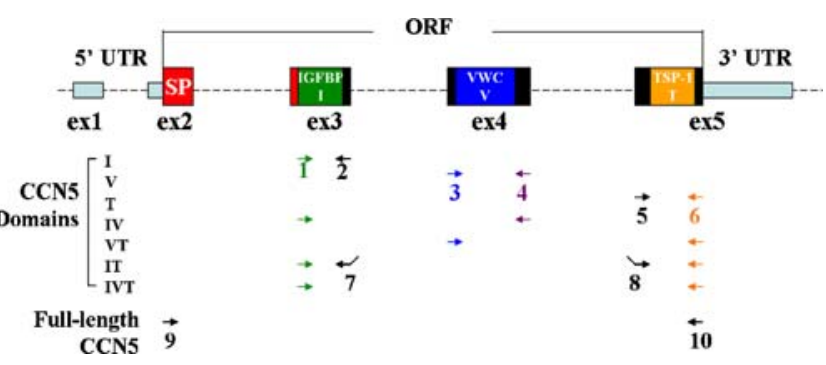

Fig. 1 Rat CCN5 domain primer design. Each primer is designated by a number, and the same color denotes identical primers. Each domain was amplified using the primer pairs shown here. Domain IT is a domain generated by joining domains I and $\mathrm{T}$ together, separated by an XhoI site linker. Full-length CCN5 amplifies the entire openreading-frame

Production and screening of anti-CCN5 positive hybridomas

Immunizations and fusions were done essentially as described (Harlow and Lane 1988; Preston et al. 2008) with some modifications. The isolated GST-fusion proteins were grouped into two groups: one group contained GST-I, GST-V, GST-T, and the other group contained GST-IV and GST-IVT. Each group was injected into two Swiss Webster and two Balb/c mice. The initial injection into the footpad of mice used 100ul 1:100 dilution of purified inclusion bodies. This was followed by four additional abdominal injections, three weeks apart, of smashed SDS-PAGE gel slices of GST-fusion protein bands. Seven days after the final injection, tail vein blood was drawn to check for anti-CCN5 positive serum. Splenocytes from serum-positive mice were dissociated and mixed with NSOI myeloma cells. A 50\% Polyethylene glycol solution (PEG1500, 50\% w/v in $75 \mathrm{mM} \mathrm{HEPES,} \mathrm{Boehringer}$ Mannheim) was added slowly to the cell pellet with gentle mixing in a $37^{\circ} \mathrm{C}$ water bath and incubated for 90 more seconds, after which warm DMEM was added drop-by-drop. Finally, cells were diluted into complete HAT media and aliquoted into 96-well plates. Cells were cultured for 10 days without changing media until visible clones had formed.

Screening of hybridomas was carried out using immunofluorescence microscopy on transfected cells grown on 24-well slides as described below. Cultured cells (either $3 \mathrm{~T} 3$ or $\mathrm{BHK}$ ) were transfected with the dual-tagged CCN5 domain constructs. Protein expression was verified by antimyc antibody (9E10, McKeon lab). Positive clones were subcloned, expanded, and frozen down in liquid nitrogen.

Purification of monoclonal antibody

Hybridoma cells were grown in B-27 media for 3 weeks. Antibodies were purified from the supernatant by affinity chromatography. Briefly, supernatant was mixed 3:1 with binding buffer (50 mM Tris- $\mathrm{Cl}, 150 \mathrm{mM} \mathrm{NaCl}, 0.05 \%$ sodium azide, $\mathrm{pH}$ 7.4) plus phenylmethylsulfonyl fluoride (PMSF) to final concentration of $0.5 \mathrm{mM}$. Protein G Sepharose 4 Fast Flow beads (GE Healthcare, 17-0618-01) were added to the solution and gently rotated at $4^{\circ} \mathrm{C}$ overnight. Protein $\mathrm{G}$ beads were packed into a column and washed twice with binding buffer plus PMSF. Pure IgG was eluted with $0.1 \mathrm{M}$ glycine, $\mathrm{pH}$ 2.7. Each $450 \mu \mathrm{l}$ of elution were collected into tubes containing $50 \mu \mathrm{l} 1 \mathrm{M}$ Tris- $\mathrm{Cl}, \mathrm{pH} 9.0$. The protein concentration of each fraction was assessed spectrophotometrically at A280. The tubes containing the highest amount of protein were combined and concentrated using the Millipore Amicon Ultra-15 centrifugal filter (Millipore, UFC901024). The concentrated antibody was then replaced into 50\% glycerol in PBS storage buffer. Protein concentration was determined by absorbance at A280 using a Nanodrop instrument (Thermo Scientific), and adjusted to $1 \mathrm{mg} / \mathrm{ml}$.

Plasmid transfection and immunofluorescence microscopy

Cells (BHK and 3T3) were transfected with Lipofectamine 2000 (Invitrogen) according to a modification of the manufacturer's protocol. Exponentially growing cells were trypsinized, re-suspended in transfection media (DMEM media with 10\% BGS and L-Glutamine), plated onto multiwell slides (Erie Scientific), and allowed to grow to 70$80 \%$ confluence. In each $9 \mathrm{~mm}$ well, $0.2 \mu \mathrm{g}$ DNA and $0.4 \mathrm{ul}$ Lipofectamine were mixed in 10ul Opti-MEM media (GIBCO) and incubate at room temperature for $20 \mathrm{~min}$, and then diluted with $150 \mathrm{ul}$ transfection media. The mixture was added onto the cells of each well. Cells were cultured with this mixture for 16-24 h, and then replaced with normal medium for another $24-48 \mathrm{~h}$.
Table 2 Primer pairs for dual-tagged rat CCN5 domain constructs and their product sizes

\begin{tabular}{lllll}
\hline Domains & Primers & PCR size (bp) & Fusion proteins & Predicated protein size (kda) \\
\hline I & $1+2$ & 228 & flag-I-myc & 10.2 \\
V & $3+4$ & 267 & flag-V-myc & 12.2 \\
T & $5+6$ & 231 & flag-T-myc & 10.7 \\
IV & $1+4$ & 483 & flag-IV-myc & 19.7 \\
VT & $3+6$ & 486 & flag-VT-myc & 20.2 \\
IT & $1+7,8+6$ & 453 & flag-IT-myc & 18.4 \\
IVT & $1+6$ & 702 & flag-IVT-myc & 27.6 \\
\hline
\end{tabular}


To screen for positive anti-CCN5 hybridomas, plasmids were transfected in $100 \mathrm{~mm}$ dishes and then trypsinized. 2$3 \times 10^{3}$ cells were aliquoted onto each well of 24 -well glass slides and cultured for additional 16-24 h. After fixation and permeabilization, slides were stored in $50 \%$ glycerol in PBS at $-20^{\circ} \mathrm{C}$. Slides can be stored in these conditions for at least 2 weeks without loss of expressed proteins. For each $100 \mathrm{~mm}$ dish, 20ug of DNA and 40ul Lipofectamine in 500ul Opti-MEM was used instead.

Transfected cells were fixed with $3 \%$ formaldehyde in PBS for 7-10 min, then permeabilized by washing three times with 1x PBST (PBS containing 0.1\% Triton X-100) for $3 \mathrm{~min}$ each. Wells were washed with PBS three times before adding primary antibody. Unless noted, primary antibodies were normally diluted 1:100 in PBS, and the secondary antibody (AlexaFluor 594 goat-anti-mouse, Invitrogen) was diluted 1:1000 in PBS. Cells were incubated with antibodies for $1 \mathrm{~h}$ at room temperature. Cell nuclei were visualized using DAPI $(1 \mu \mathrm{g} / \mathrm{ml}$ in PBS for $5 \mathrm{~min}$ ). All washing steps between incubations were done 3 times with PBS for 5 min each, unless otherwise noted. Slides were mounted with $90 \%$ Glycerol in $10 \mathrm{mM}$ Tris-Cl, $\mathrm{pH}$ 7.4. Pictures were taken on a Carl Zeiss Axio fluorescent microscope and analyzed by AxioVersion software.

\section{Western Blot analysis}

We followed the protocol previously described (Lake et al. 2003). Briefly, cultured cells were washed twice with cold PBS for 5 min each, then lysed with RIPA buffer (1\% NP$40,0.5 \%$ sodium deoxycholate, $0.1 \%$ SDS, $0.15 \mathrm{M} \mathrm{NaCl}$, $0.05 \mathrm{M}$ Tris-HCl, $\mathrm{pH} 7.5)$ plus $1: 50$ protease inhibitor (Sigma, P8340). Proteins were denatured at $95^{\circ} \mathrm{C}$ for $5 \mathrm{~min}$ with $2 \mathrm{X}$ loading buffer (100 mM Tris-Cl, pH6.8, 4\% SDS, $20 \%$ glycerol, $200 \mathrm{mM}$ DTT, $0.2 \%$ Bromophenol Blue), and then separated by $12 \%$ SDS-PAGE and transferred onto 0.2 $\mu \mathrm{m}$ pore size Immuno-Blot polyvinylidene difluoride membranes (PVDF, Bio-Rad). Dried membranes were rewetted and blocked with $5 \%$ non-fat milk in TBST $(137 \mathrm{mM} \mathrm{NaCl}$, $20 \mathrm{mM}$ Tris, $\mathrm{pH} 7.6,0.2 \%$ Tween 20). The purified antibody 22H10 was diluted 1:2000 in milk-TBST; other antibodies were diluted 1:1000 in milk-TBST; horseradish peroxidase (HRP)-conjugated secondary antibody anti-mouse or antirabbit IgG (Santa Cruz) was diluted 1:5000. All washing steps were done three times with PBST for 10 min each. Membranes were developed with ECL reagent (GE-Amersham, RPN2132), and exposed with X-Omat Blue XB-1 Film (Kodak).

\section{Immunoprecipitation}

Cell lysates were prepared as described above for Western Blot analysis. Primary antibodies $(22 \mathrm{H} 10$ and control mouse $\operatorname{IgG}$ ) and cell lysates was incubated overnight at $4^{\circ} \mathrm{C}$ with gentle agitation then incubated with Protein $\mathrm{G}$ Sepharose 4 Fast Flow beads for $1 \mathrm{~h}$ at room temperature. The protein-antibody-bead complex was spun down at $2000 \times \mathrm{g}$ for $2 \mathrm{~min}$, and the resulting pellet was washed six times with RIPA buffer containing protease inhibitor. Finally, the protein complex was denatured in loading buffer at $95^{\circ} \mathrm{C}$ for $2 \mathrm{~min}$ and analyzed by Western Blot. Membranes were stained with Ponceau S staining $(0.2 \%$ Ponceau S, 1\% acetic acid) to visualize proteins.

Immunofluorescence microscopy with Dylight 594-labeled primary antibodies

Equal amounts of purified 22H10 and control mouse IgG (Bethyl Laboratories) were directly conjugated with Dylight 594 fluorescent dye using the Dylight Antibody Labeling Kit (Pierce). After labeling and purification, protein and dye incorporation were determined using a Nanodrop instrument to ensure equal application of $22 \mathrm{H} 10$ and control mouse IgGs on tissue sections.

The immunofluorescence microscopy protocol and paraffin-embedded rat intestine sections were kindly provided by Wen-Chi Yang in Dr. McKeon's laboratory at Harvard Medical School. Briefly, small intestine and colon of 6-8 week old female rats were isolated and fixed overnight in 3.7\% formaldehyde at $4{ }^{\circ} \mathrm{C}$, then put into $70 \%$ ethanol until use. Tissues were paraffin embedded and sectioned at the Harvard Rodent Histological Center. Sections were de-paraffined and re-hydrated through xylene, 100\% ethanol, $95 \%$ ethanol, and PBS. Antigen retrieval was done by immersing the slides in $10 \mathrm{mM}$ citrate for $15-20 \mathrm{~min}$ at $92.8^{\circ} \mathrm{C}$, followed by slow cooling to room temperature before transfer into PBS. Sections were blocked for $30 \mathrm{~min}$ at room temperature with PBS containing 10\% BSA and 1:100 normal mouse serum. Dylight 594 labeled primary antibodies were added at different dilutions in PBS onto each section, and incubated overnight at $4^{\circ} \mathrm{C}$. Slides were washed with PBST (PBS with $0.1 \%$ tween20) three times for $5 \mathrm{~min}$ each. Cell nuclei were visualized using DAPI ( $1 \mu \mathrm{g} / \mathrm{ml}$ in PBS for $5 \mathrm{~min})$. Slides were mounted using 90\% Glycerol in $10 \mathrm{mM}$ Tris-Cl, $\mathrm{pH} 7.4$. Pictures were taken using a Zeiss Axio fluorescence microscope and analyzed by AxioVersion software.

\section{Results}

Production and screening of anti-CCN5 monoclonal antibodies

To obtain the monoclonal antibodies against each domain of rat CCN5, we first expressed rat CCN5 domains as GSTfusion proteins in the bacterial strain BL21. After IPTG 
induction at $37^{\circ} \mathrm{C}$, the fusion proteins were expressed as inclusion bodies and isolated by sonication and centrifugation. Following SDS-PAGE, Coomassie staining (Fig. 2) revealed that the dominant bands appeared at the predicted sizes of the fusion proteins, and were confirmed to be CCN5 using a specific rabbit anti-CCN5 polyclonal antibody, NGRR, raised against a peptide fragment of the rat CCN5 VWC domain NGRRYLDGETFKPNC, same region as human CCN5 previously described (Gray et al. 2007; Jones et al. 2007; Lake et al. 2003; Mason et al. 2004a). The additional bands visible in the Western blot are the result of insufficient reduction of compacted proteins in the inclusion bodies.

Mice were immunized with a mixture of GST-fused CCN5 domain proteins as described in Material and Methods. Swiss Webster mice were more immunogenic than Balb/c mice, with 3 out of 4 positive for anti-CCN5 antibodies after four injections compared with 1 out of 4 testing positive for production of CCN5 antibodies (data not shown). In addition, and not unexpectedly, we found that the larger proteins were more immunogenic than smaller proteins: only 1 of the 4 mice injected with a mixture of GST fusion peptides linked to the individual I, $\mathrm{V}$, and $\mathrm{T}$ domains were positive for anti-CCN5 antibodies, whereas 3 of 4 mice injected with a mixture of GST fusion peptides linked with the IV and IVT domains produced anti-CCN5 antibodies (not shown).

Two of the Swiss Webster mice that were positive for anti-CCN5 antibodies were sacrificed and their spleen cells fused with myeloma cells. After selection in HAT media for 10 days, single hybridoma clones were visible and the supernatant from wells containing single clones was combined and screened as described in Material and Methods. We used immunofluorescence microscopy of BHK cells transfected with plasmids expressing dualtagged domain proteins to screen monoclonal antibodies, thus eliminating the many false positives against GST seen in screening methods using Western Blot analysis with the GST-fusion protein.

Fig. 2 Purified inclusion bodies of BL21 express GST-fused rat CCN5 domains. a Coommassie staining of samples run on reducing SDS-PAGE gels. b Western Blot analysis using the polyclonal anti-CCN5 antibody NGRR, raised against a peptide from V-domain of rat CCN5
All identified monoclonal antibodies are against the V-domain of CCN5

Following two rounds of screening, 32 clones were identified that produced anti-CCN5 antibodies. Further mapping of the antibodies' targeted epitopes on CCN5 by Western blot using GST-fused CCN5 domain proteins showed that all clones produced antibodies against the $\mathrm{V}$ domain of CCN5 (not shown). We picked one clone, $22 \mathrm{H} 10$, with a particularly high antibody titer among the hybridoma clones, for expansion, purification, and further characterization. All other clones were placed into liquid nitrogen storage for future analysis and use.

Antibody $22 \mathrm{H} 10$ characterization demonstrates that it recognizes the $\mathrm{V}$-domain

To isolate purified $22 \mathrm{H} 10$ antibody, hybridoma clone $22 \mathrm{H} 10$ was grown in B-27 supplemented DMEM for 3 weeks to produce maximum antibody titer, purified by protein G-conjugated Sepharose beads, and subjected to SDS-PAGE analysis (Fig. 3). To determine which epitope the antibody was directed against, we carried out both immunofluorescence microscopy and Western Blot analysis of purified 22H10 antibody (Fig. 4). The antibody recognized all dual-tagged rat $\mathrm{CCN} 5$ domain constructs containing the $\mathrm{V}$ domain of $\mathrm{CCN} 5$, but none of the constructs without the $\mathrm{V}$ domain. Expression of all duallabeled domains was confirmed by both anti-Flag and antiMyc antibody staining and Western Blot analysis (Fig. 4). These data indicate that antibody $22 \mathrm{H} 10$ is directed against the V-domain of CCN5.

22H10 immunoprecipitates recombinant and endogenous CCN5 protein

Having determined that the $22 \mathrm{H} 10$ antibody could detect the V-domain of CCN5 in both immunofluorescence and Western Blot analyses, we assessed whether or not the

\section{Commassie Staining}

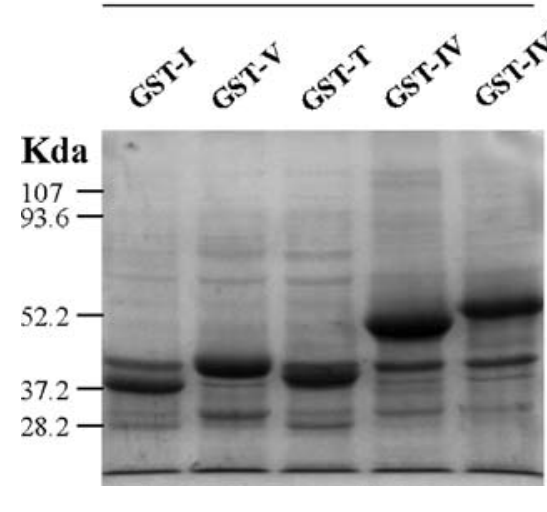

IB: NGRR (anti-CCN5 pAb)

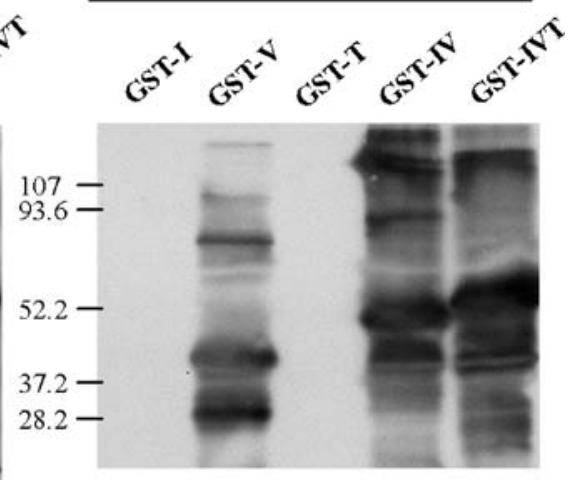


Fig. 3 Coommassie staining of clone $22 \mathrm{H} 10$ was grown in B-27 supplement media for 3 weeks. Cells were lysed and the lysate applied to a Protein G-conjugated agarose column. Bound material was eluted from the column and run on $12 \%$ reduced SDS-PAGE. The upper band is IgG heavy chain $(\sim 50 \mathrm{Kda})$, and lower band is light chain $(\sim 24 \mathrm{Kda})$ purified 22 h10. Hybridoma

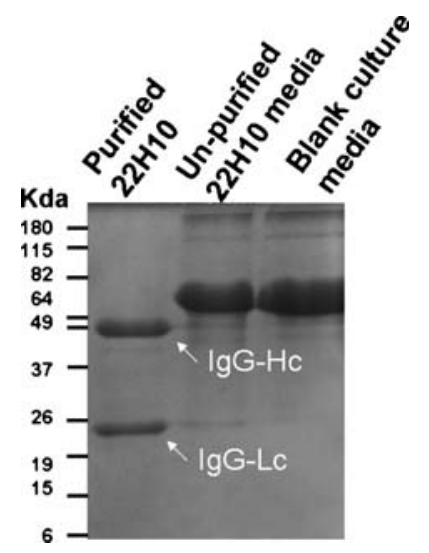

Compared to mouse IgG controls, 22H10 antibody stained strongly for rat intestine epithelium. Other cell types had less staining compare to epithelium (Fig. 7). Interestingly, at higher magnification, the nuclear staining of $22 \mathrm{H} 10$ in smooth muscle cells and fibroblasts are readily apparent compared to mouse IgG controls. Similar results were seen in rat colon (data not shown). This result indicates that $22 \mathrm{H} 10$ displays the same expression pattern in mouse as other anti-CCN5 antibodies previously used in embryos (Jones et al. 2007) and adult mice (Gray et al. 2007).

\section{Discussion}

antibody could immunoprecipitate CCN5 protein, as this would be a potentially useful characteristic for future studies. Compared to the mouse IgG control, 22H10 was able to recognize both recombinant and endogenous CCN5 (Fig. 5a). It was also able to precipitate recombinant CCN5 from lysates of $3 \mathrm{~T} 3$ cells expressing flag-IVT-myc protein (Fig. 5b), and endogenous CCN5 from growth arrested rat aortic smooth muscle cells (Fig. 5c). Mouse IgG controls neither recognized nor precipitated CCN5. The previously described rabbit polyclonal anti-CCN5 peptide antibody NGRR confirmed the expression of both recombinant (Fig. 4b) and endogenous CCN5 (Fig. 5c).

\section{$22 \mathrm{H} 10$ reacts with mouse and rat CCN5, but not human CCN5}

To test the specificity of $22 \mathrm{H} 10$ antibody on different species, we cloned human, mouse and rat CCN5 into the Gateway expression vector. V5 and 6XHis tags were included in C-terminal of the recombinant protein. The human and mouse sequences were cloned from the Integrated Molecular Analysis of Genomes and their Expression (IMAGE) Consortium clones distributed by Open Biosystems (http://image.hudsonalpha.org/), while the rat CCN5 sequence was cloned from growth arrested SDSM cells as described in Materials and Methods. All plasmids were sequenced for identity and accuracy before using them in the cloning procedure. $\beta$-galactosidase was cloned and used as a negative control. 22H10 antibody recognized rat and mouse CCN5 recombinant proteins, but not human CCN5, in both immunofluorescence (Fig. 6a) and Western Blot analyses (Fig. 6b). As expected, LacZ was not recognized by $22 \mathrm{H} 10$ antibody.

\section{H10 detects endogenous mouse CCN5 in tissues}

The ability of $22 \mathrm{H} 10$ antibody to recognize CCN5 in tissues was assessed by direct application of Dylight 594 conjugated primary antibodies to rat intestine sections.
In this paper, we report the generation of monoclonal antibodies directed against specific domains of the CCN5 protein. A total of 32 monoclonal antibodies against rat CCN5 were generated in this study. Although they are all against $\mathrm{V}$ domain of CCN5, they require further characterization to determine their relative specificity and uses. The possibility that one or more of the clones might be producing function-blocking antibodies remains intriguing, as does the possibility that some of the antibodies may react with human CCN5. Many of the other reagents generated in this study should be useful tools as well. The dual-tagged CCN5 domain constructs may be used in studies of isoforms and domains of CCN5. The GST-fused proteins vitro and in vivo.

One useful technical feature of the monoclonal antibody process we designed is the use of immunofluorescence microscopy of transfected BHK cells grown on 24-well glass slides to detect anti-CCN5 positive hybridoma clones. This proved to be much more efficient in terms of both time and labor compared to the standard approaches that use ELISA or Western Blot analysis to screen hybridoma clones. Using the preparation and preservation conditions described in Materials and Methods, samples can be stored for at least 2 weeks.

We purified and characterized one anti-CCN5 monoclonal antibody, $22 \mathrm{H} 10$, and found that it is specific for the $\mathrm{V}$-domain. It recognizes and reacts with mouse and rat CCN5 and V-domains, but not human. This species specificity profile suggests that it will be useful in humanmouse grafts to distinguish the mouse cells from the human. Importantly, we demonstrate that $22 \mathrm{H} 10$ recognizes and reacts with endogenous CCN5 as well recombinant protein. Since $22 \mathrm{H} 10$ can immunoprecipitate as well as identify CCN5, it may be useful in identifying CCN5 binding partners and other interacting proteins.

Though we designed the monoclonal antibody generation to produce antibodies against each domain of CCN5 by immunizing mice with a mixture of GST-fused domains, we have applications in the study of CCN5 function both in 
Fig. 4 22H10 recognizes the V-domain of CCN5. Rat CCN5 domains are expressed by transfected plasmids in 3T3 cells. All proteins are tagged with flag at $\mathrm{N}$-terminal and c-myc at the C-terminal. Labels are 0 mock, 1 flag-I-myc, 2 flag-V-myc, 3 flag-T-myc, 4 flag-IV-myc, 5 flag-VT-myc, 6 flag-IT-myc, 7 flag-IVT-myc $A$. proteins detected by immunofluorescence microscopy. Each antibody stained separately, DAPI shows the typical cell density. $B$. expressed dual-tagged domain proteins are detected by Western blot. Lysates of each domain construct transfected 3T3 cells are indicated as each lane. Note a cloning error make I domain do not have c-myc tag, hence not detected by anti-myc antibody a

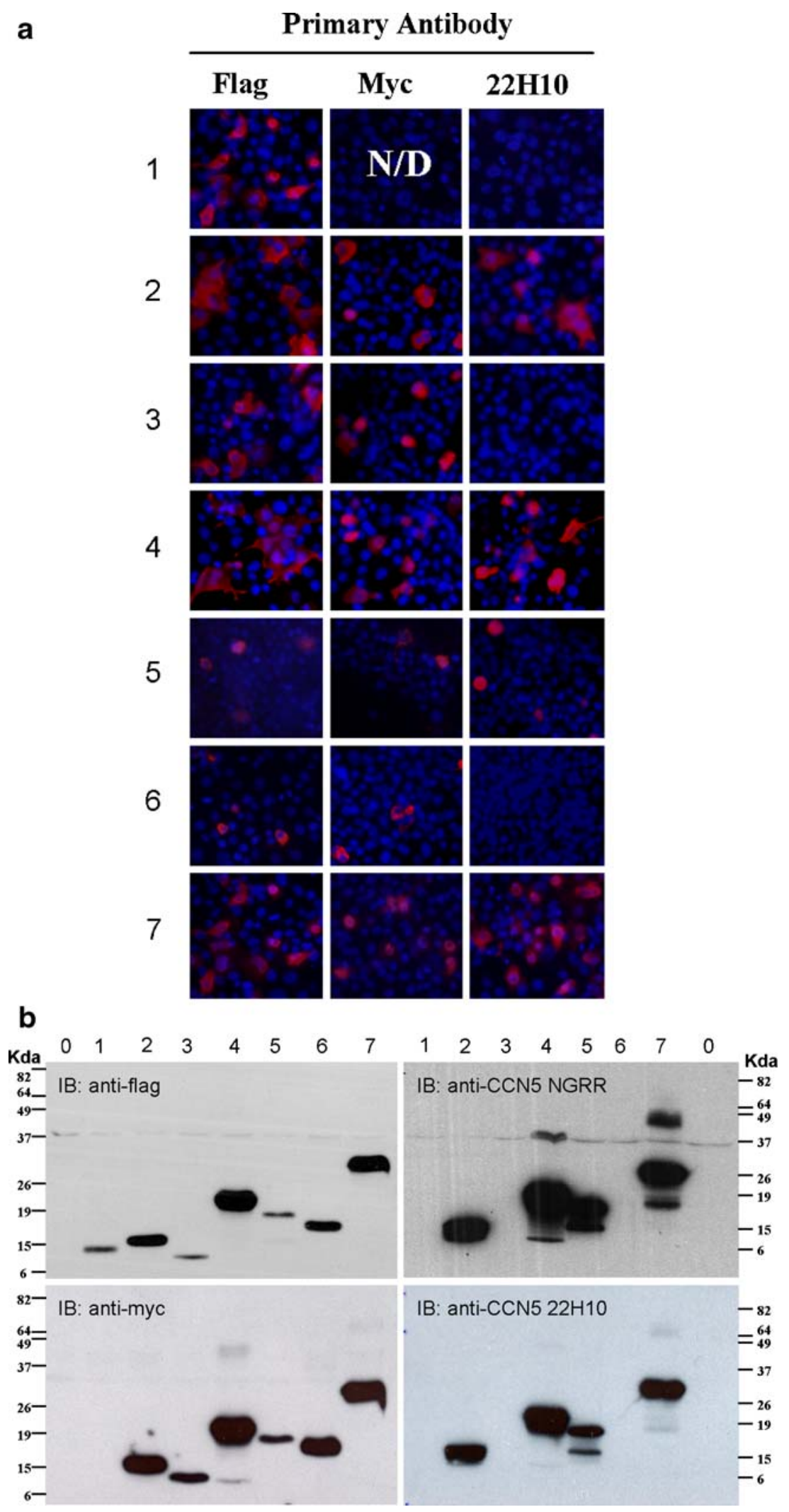

b 
Fig. 5 22H10 recognizes and immunoprecipitates recombinant $\mathrm{CCN} 5$ and endogenous CCN5. Mouse IgG serves as control for immunoprecipitation and western blot. GAPDH and Ponceau staining show the equal loading of proteins. A. $22 \mathrm{H} 10$ recognizes flag-IVT-myc and CCN5 in growth arrested SDSM cells. B. 3 T3 cells were transfected with plasmid expressing flag-IVT-myc for $48 \mathrm{~h}$. Cells were lysed and immunoprecipitated with $22 \mathrm{H} 10$ and detected using anti-myc antibody. Immuno-complexes were dissociated in reducing loading buffer and run on $12 \%$ SDS-PAGE gel. $C$. SDSM cells were cultured using conditions that rendered them quiescent $\left(\mathrm{G}_{0}\right)$ or allowed them to proliferate exponentially (Exp). Cells were lysed and immunoprecipitated with $22 \mathrm{H} 10$, and $\mathrm{CCN} 5$ was detected using NGRR antibody a 3T3 cells transfected with flag-IVT-myc construct

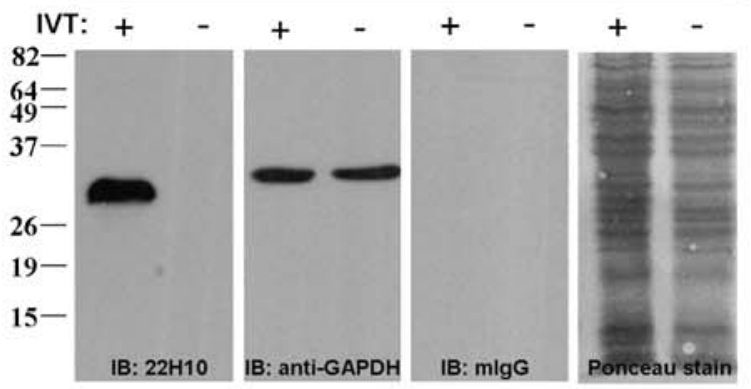

rat aortic smooth muscle cells

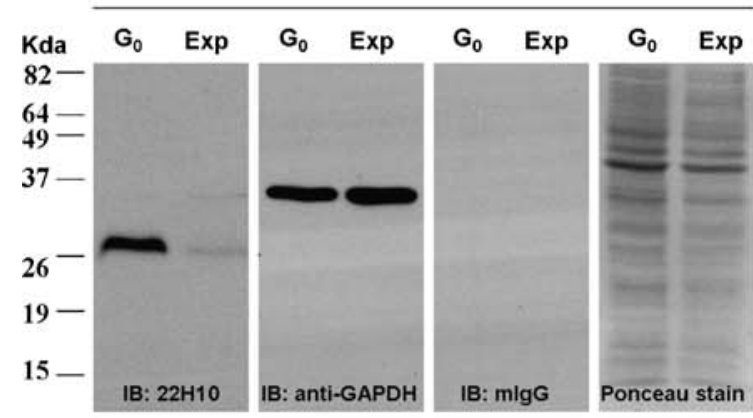

b

IB: anti-myc

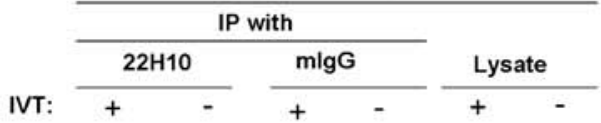

$\frac{\text { Ponceau stain }}{\text { IP with }}$
$\frac{22 \mathrm{H} 10}{+\quad}$
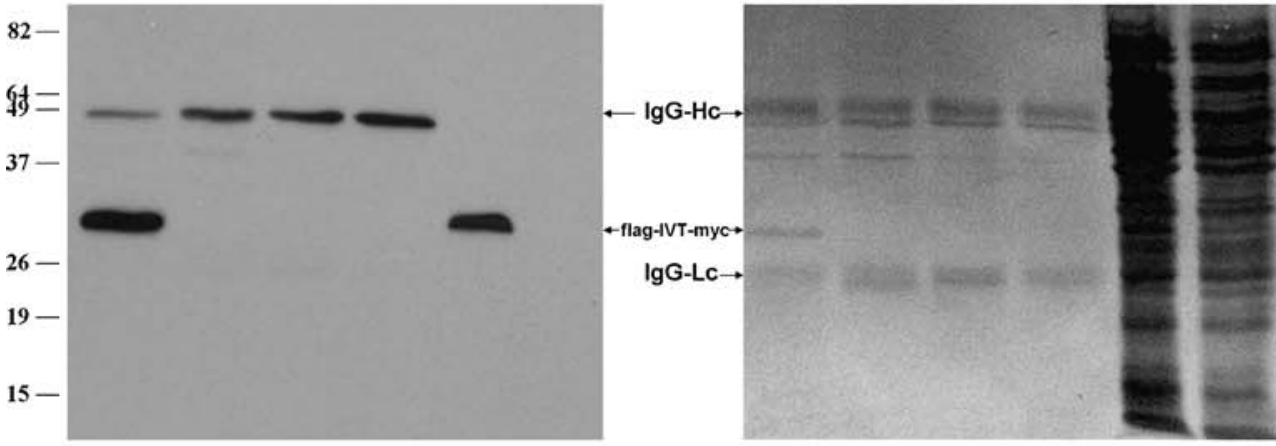

C

IB: NGRR
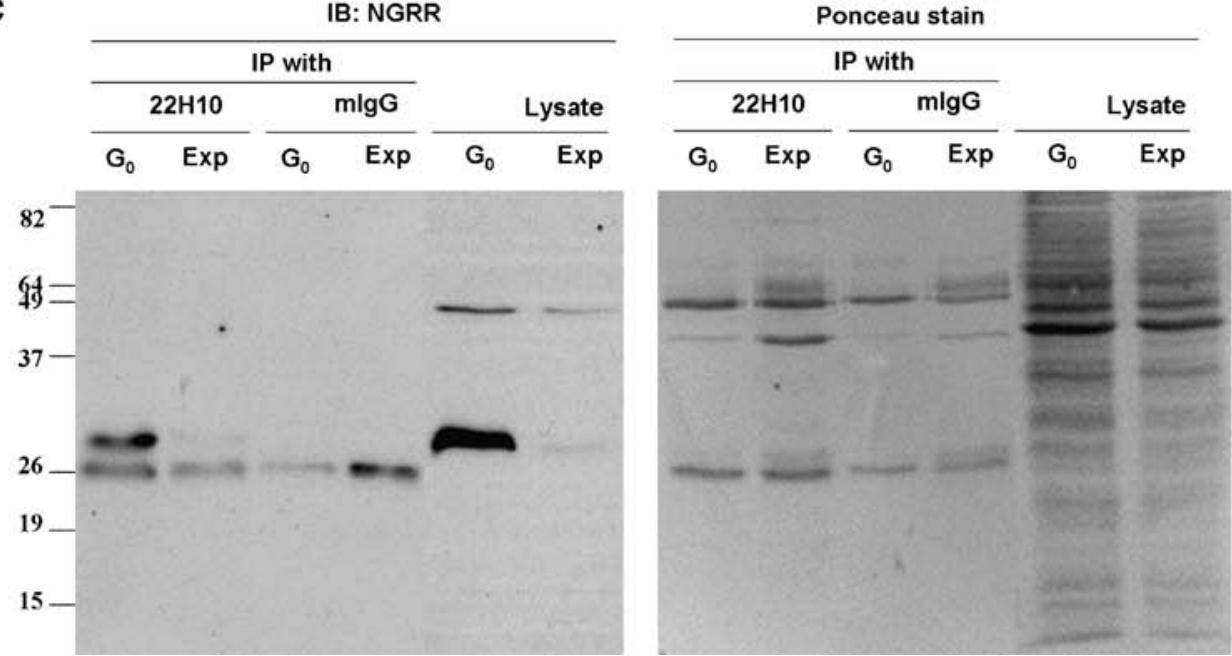
Fig. $622 \mathrm{H} 10$ reacts with mouse and rat CCN5, but not with human recombinant $\mathrm{CCN} 5$. 3 T3 cells were transfected with plasmids that express V5-tagged CCN5 of the indicated species. $A$. Immunofluorescence microscopy of $3 \mathrm{~T} 3$ cells. B. 3T3 cell lysates were subjected to Western Blot analysis. 22H10 recognizes mouse and rat, but not human recombinant CCN5 a
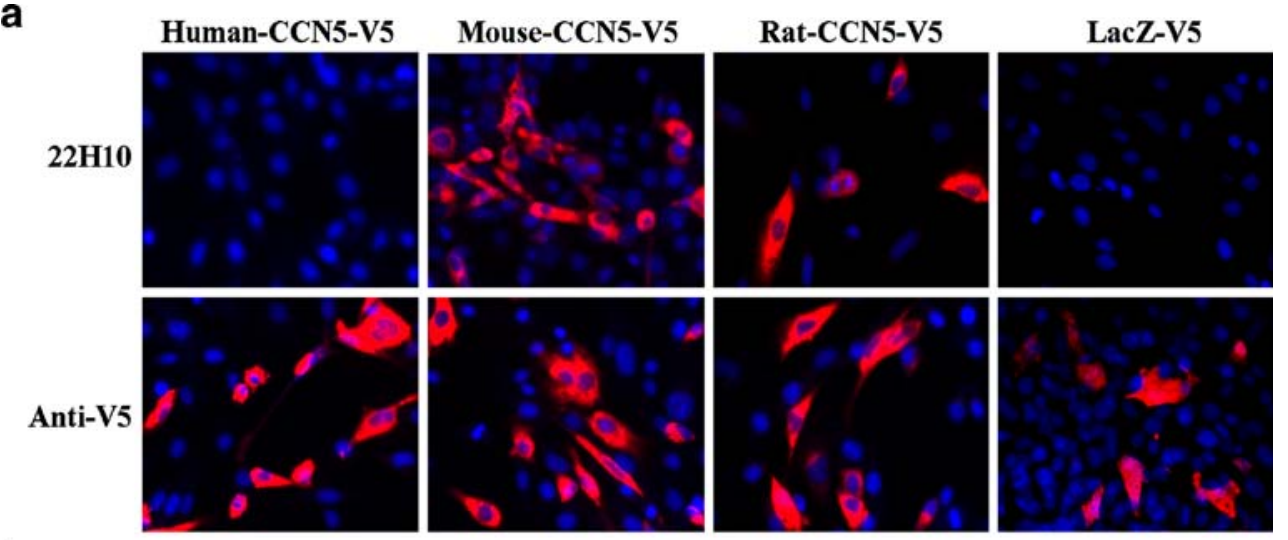

b

rCCN5-V5

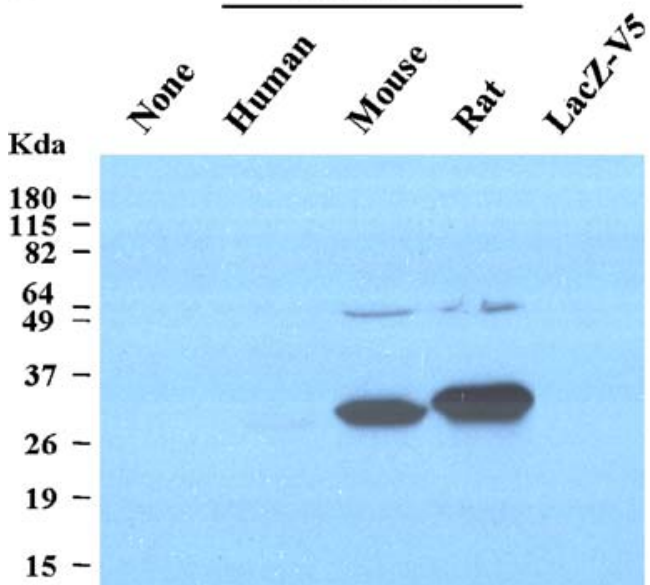

IB: $22 \mathrm{H} 10$

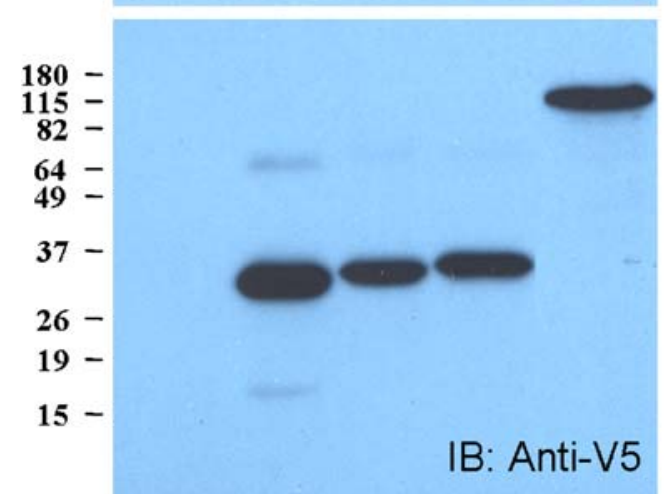

did not detect the spectrum of antibodies against each domain of CCN5 as we had anticipated. Instead, all of the antibodies are against epitopes in the V-domain of CCN5. This suggests that the $\mathrm{V}$-domain is more immunogenic than the other domains. Of note is that when the CCN5 amino acid sequence is analyzed to reveal the most immunogenic regions, a 14 amino acid sequence in the V-domain was identified, and that is what we used to generate rabbit polyclonal anti-CCN5 antibody NGRR. This is not entirely unexpected: the immunogenicity of the V-domain has also been demonstrated in other $\mathrm{CCN}$ family members. For example, in CCN2, computer-assisted prediction of epitome immunogenicity indicates that the V-domain and CT domain (which is absent in CCN5) are the most highly immunogenic (Minato et al. 2004). Further, injection of full-length recombinant CCN2 into mouse and rabbits, the majority of antibodies generated recognized the $\mathrm{V}$-domain of CCN2 (Minato et al. 2004). Future efforts to produce domain-specific monoclonal antibodies are much more likely to be successful if only the I-domain, T-domain, or amino acid sequences contained within these domains, are used as antigens. Nor is the monoclonal approach to antibody generation the only viable option. By injecting the domain peptides separately, Perbal and colleagues 

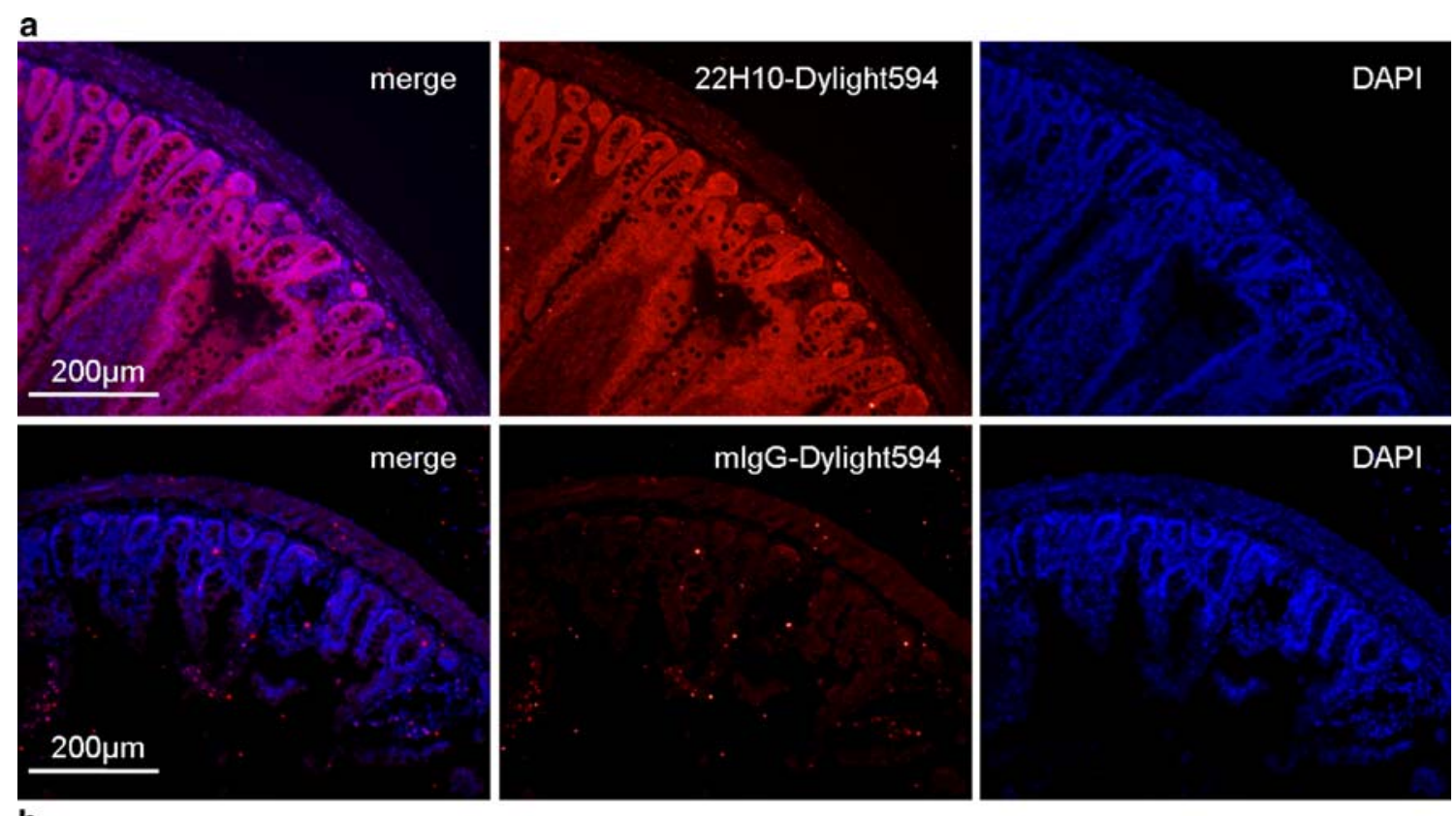

b
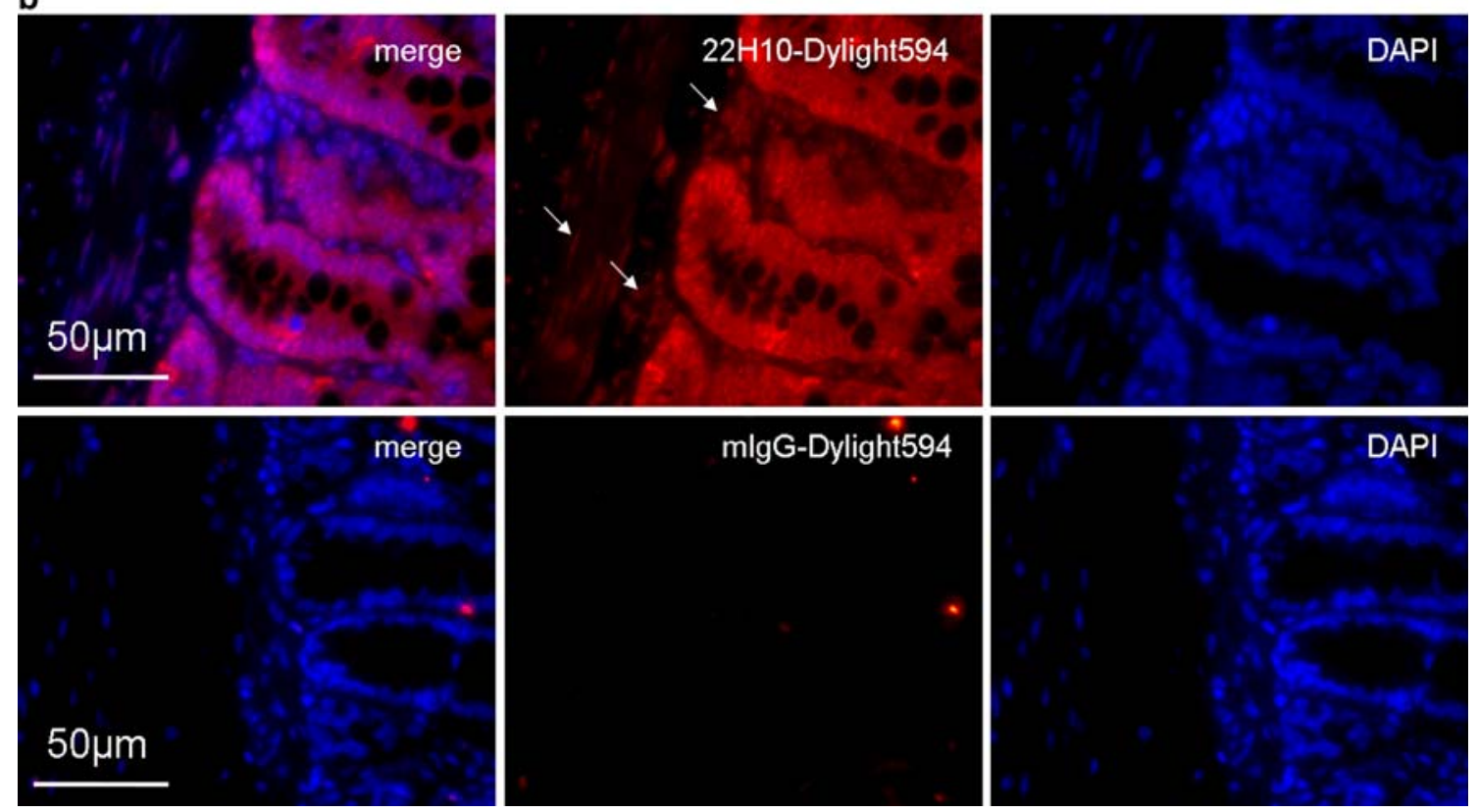

Fig. 7 22H10 recognizes CCN5 in vivo. Immunofluorescence microscopy using $22 \mathrm{H} 10$ directly conjugated to Dylight 594 fluorescent dye was carried out on paraffin embedded rat intestine cross sections. Sections are blocked using PBS containing 10\% BSA and 1:100 normal mouse serum. Dylight 594 labeled antibodies are diluted 1:100 in PBS.
$A$. Lower magnification shows the structure of intestine and the strong staining of the epithelium by $22 \mathrm{H} 10$. B. At higher magnification, nuclear staining was more apparent in other cell types. This staining pattern in rat GI track is the same as another anti-CCN5 antibody (NGRR) previously reported in mouse embryo and adult tissues succeeded in generating rabbit polyclonal antibodies against each domain of CCN3 (Lazar et al. 2007), and this remains a viable approach to $\mathrm{CCN} 5$ domain analysis as well.

We used direct dye labeling of primary antibodies and application to tissue sections. This method eliminates non-specific signals caused by endogenous peroxidase and non-specific binding of anti-mouse secondary antibody.
Although this approach is not as sensitive as immunohistochemistry and indirect immunofluorescence, we were able to visualize CCN5 staining quite easily in tissue sections with direct-labeled 22H10. Taken together, the data presented in this communication on the characterization of $22 \mathrm{H} 10$ indicates that it is a highly specific monoclonal antibody that will be useful for a variety of applications in the study of CCN5. 
Acknowledgements We thank Richard Krolewski (Program in Cell, Molecular and Developmental Biology, Tufts Sackler School) and Wen-Chi Yang (Harvard Medical School) for their assistance with the tissue section staining. In addition, we thank Ronald Myers, Kristina Cvitanovic, and Cassandra Baughman (Program in Cell, Molecular and Developmental Biology, Tufts Sackler School) for their critical reading of, and discussions about, the manuscript. This work was supported in part by grants HL049973 and HD046251 from the National Institutes of Health.

Open Access This article is distributed under the terms of the Creative Commons Attribution Noncommercial License which permits any noncommercial use, distribution, and reproduction in any medium, provided the original author(s) and source are credited.

\section{References}

Banerjee S, Dhar G, Haque I, Kambhampati S, Mehta S, Sengupta K, Tawfik O, Phillips TA, Banerjee SK (2008) CCN5/WISP-2 expression in breast adenocarcinoma is associated with less frequent progression of the disease and suppresses the invasive phenotypes of tumor cells. Cancer Res 68:7606-7612. doi:10.1158/0008-5472.CAN-08-1461

Bleau AM, Planque N, Perbal B (2005) CCN proteins and cancer: two to tango. Front Biosci 10:998-1009. doi:10.2741/1594

Brigstock DR, Steffen CL, Kim GY, Vegunta RK, Diehl JR, Harding PA (1997) Purification and characterization of novel heparinbinding growth factors in uterine secretory fluids. Identification as heparin-regulated $\mathrm{Mr} \mathrm{10,000} \mathrm{forms} \mathrm{of} \mathrm{connective} \mathrm{tissue}$ growth factor. J Biol Chem 272:20275-20282. doi:10.1074/ jbc.272.32.20275

Chen CC, Lau LF (2008) Functions and mechanisms of action of $\mathrm{CCN}$ matricellular proteins. Int J Biochem Cell Biol

Chen N, Leu SJ, Todorovic V, Lam SC, Lau LF (2004) Identification of a novel integrin alphavbeta3 binding site in CCN1 (CYR61) critical for pro-angiogenic activities in vascular endothelial cells. J Biol Chem 279:44166-44176. doi:10.1074/jbc.M406813200

Chevalier G, Yeger H, Martinerie C, Laurent M, Alami J, Schofield PN, Perbal B (1998) novH: differential expression in developing kidney and Wilm's tumors. Am J Pathol 152:1563-1575

de Winter P, Leoni P, Abraham D (2008) Connective tissue growth factor: structure-function relationships of a mosaic, multifunctional protein. Growth Factors 26:80-91. doi:10.1080/ 08977190802025602

Delmolino LM, Stearns NA, Castellot JJ Jr (2001) COP-1, a member of the CCN family, is a heparin-induced growth arrest specific gene in vascular smooth muscle cells. J Cell Physiol 188:45-55. doi: $10.1002 /$ jcp. 1100

Gray MR, Malmquist JA, Sullivan M, Blea M, Castellot JJ Jr (2007) CCN5 Expression in mammals. II. Adult rodent tissues. J Cell Commun Signal 1:145-158. doi:10.1007/s12079-007-0013-z

Harlow E, Lane D (1988) Antibodies: A Laboratory Manual. Cold Spring Harbor, New York

Holbourn KP, Acharya KR, Perbal B (2008) The CCN family of proteins: structure-function relationships. Trends Biochem Sci 33:461-473. doi:10.1016/j.tibs.2008.07.006

Jones JA, Gray MR, Oliveira BE, Koch M, Castellot JJ Jr (2007) CCN5 expression in mammals : I. Embryonic and fetal tissues of mouse and human. J Cell Commun Signal 1:127-143. doi:10.1007/s12079-007-0012-0

Katsuki Y, Sakamoto K, Minamizato T, Makino H, Umezawa A, Ikeda MA, Perbal B, Amagasa T, Yamaguchi A, Katsube K (2008) Inhibitory effect of CT domain of $\mathrm{CCN} 3 / \mathrm{NOV}$ on proliferation and differentiation of osteogenic mesenchymal stem cells, Kusa-A1. Biochem Biophys Res Commun 368:808-814. doi:10.1016/j.bbrc.2008.02.010

Kubota S, Takigawa M (2007) CCN family proteins and angiogenesis: from embryo to adulthood. Angiogenesis 10:1-11. doi:10.1007/ s10456-006-9058-5

Kutz WE, Gong Y, Warman ML (2005) WISP3, the gene responsible for the human skeletal disease progressive pseudorheumatoid dysplasia, is not essential for skeletal function in mice. Mol Cell Biol 25:414-421. doi:10.1128/MCB.25.1.414-421.2005

Lake AC, Bialik A, Walsh K, Castellot JJ Jr (2003) CCN5 is a growth arrest-specific gene that regulates smooth muscle cell proliferation and motility. Am J Pathol 162:219-231

Lake AC, Castellot JJ Jr (2003) CCN5 modulates the antiproliferative effect of heparin and regulates cell motility in vascular smooth muscle cells. Cell Commun Signal 1:5. doi:10.1186/1478-811X-1-5

Lazar N, Manara C, Navarro S, Bleau AM, Llombart-Bosch A, Scotlandi K, Planque N, Perbal B (2007) Domain-specific $\mathrm{CCN} 3$ antibodies as unique tools for structural and functional studies. J Cell Commun Signal 1:91-102. doi:10.1007/s12079007-0009-8

Leu SJ, Chen N, Chen CC, Todorovic V, Bai T, Juric V, Liu Y, Yan G, Lam SC, Lau LF (2004) Targeted mutagenesis of the angiogenic protein CCN1 (CYR61). Selective inactivation of integrin alpha6beta1-heparan sulfate proteoglycan coreceptor-mediated cellular functions. J Biol Chem 279:44177-44187. doi:10.1074/ jbc.M407850200

Leu SJ, Liu Y, Chen N, Chen CC, Lam SC, Lau LF (2003) Identification of a novel integrin alpha 6 beta 1 binding site in the angiogenic inducer CCN1 (CYR61). J Biol Chem 278:33801-33808. doi:10.1074/jbc.M305862200

Mason HR, Grove-Strawser D, Rubin BS, Nowak RA, Castellot JJ Jr (2004a) Estrogen induces CCN5 expression in the rat uterus in vivo. Endocrinology 145:976-982. doi:10.1210/en.2003-0823

Mason HR, Lake AC, Wubben JE, Nowak RA, Castellot JJ Jr (2004b) The growth arrest-specific gene CCN5 is deficient in human leiomyomas and inhibits the proliferation and motility of cultured human uterine smooth muscle cells. Mol Hum Reprod 10:181187. doi: $10.1093 / \mathrm{molehr} / \mathrm{gah} 028$

Minato M, Kubota S, Kawaki H, Nishida T, Miyauchi A, Hanagata H, Nakanishi T, Takano-Yamamoto T, Takigawa M (2004) Modulespecific antibodies against human connective tissue growth factor: utility for structural and functional analysis of the factor as related to chondrocytes. J Biochem 135:347-354. doi:10.1093/jb/mvh042

Pennica D, Swanson TA, Welsh JW, Roy MA, Lawrence DA, Lee J, Brush J, Taneyhill LA, Deuel B, Lew M, Watanabe C, Cohen RL, Melhem MF, Finley GG, Quirke P, Goddard AD, Hillan KJ, Gurney AL, Botstein D, Levine AJ (1998) WISP genes are members of the connective tissue growth factor family that are up-regulated in wnt-1-transformed cells and aberrantly expressed in human colon tumors. Proc Natl Acad Sci USA 95:1471714722. doi:10.1073/pnas.95.25.14717

Perbal B (2004) CCN proteins: multifunctional signaling regulators. Lancet 363:62-64. doi:10.1016/S0140-6736(03)15172-0

Preston A, Fodey T, Douglas A, Elliott CT (2008) Monoclonal antibody development for acrylamide-adducted human haemoglobin; A biomarker of dietary acrylamide exposure. J Immunol Methods

Rachfal AW, Brigstock DR (2005) Structural and functional properties of CCN proteins. Vitam Horm 70:69-103. doi:10.1016/S00836729(05)70003-0

Shi ZX, He F, Wang LL, Liang YM, Han H, Wang CZ, Zhao Q, Geng XD (2008) Expression, refolding, and purification of a truncated human Delta-like1, a ligand of Notch receptors. Protein Expr Purif 59:242-248. doi:10.1016/j.pep.2008.02.004

Steffen CL, Ball-Mirth DK, Harding PA, Bhattacharyya N, Pillai S, Brigstock DR (1998) Characterization of cell-associated and 
soluble forms of connective tissue growth factor (CTGF) produced by fibroblast cells in vitro. Growth Factors 15:199-213

Subramaniam MM, Lazar N, Navarro S, Perbal B, Llombart-Bosch A (2008) Expression of CCN3 protein in human Wilms' tumors: immunohistochemical detection of $\mathrm{CCN} 3$ variants using domainspecific antibodies. Virchows Arch 452:33-39. doi:10.1007/ s00428-007-0523-3

Tamatani T, Kobayashi H, Tezuka K, Sakamoto S, Suzuki K, Nakanishi T, Takigawa M, Miyano T (1998) Establishment of the enzymelinked immunosorbent assay for connective tissue growth factor (CTGF) and its detection in the sera of biliary atresia. Biochem Biophys Res Commun 251:748-752. doi:10.1006/bbrc.1998.9543
Yang GP, Lau LF (1991) Cyr61, product of a growth factor-inducible immediate early gene, is associated with the extracellular matrix and the cell surface. Cell Growth Differ 2:351-357

Zhang R, Averboukh L, Zhu W, Zhang H, Jo H, Dempsey PJ, Coffey RJ, Pardee AB, Liang P (1998) Identification of rCop-1, a new member of the CCN protein family, as a negative regulator for cell transformation. Mol Cell Biol 18:6131-6141

Zoubine MN, Banerjee S, Saxena NK, Campbell DR, Banerjee SK (2001) WISP-2: a serum-inducible gene differentially expressed in human normal breast epithelial cells and in MCF-7 breast tumor cells. Biochem Biophys Res Commun 282:421-425. doi:10.1006/bbrc.2001.4584 